To be presented at the 296th Meeting of the American Institute of Electrical Engineers, Pittsfield,

Mass., May 29, 1914.

Copyright 1914. By A. I. E. E.

(Subject to final revision for the Transactions.)

\title{
RELATIVE MERITS OF Y AND DELTA CONNECTION FOR ALTERNATORS
}

\author{
BY T. S. EDEN
}

\section{Abstract of Paper}

The main advantages obtained with the $\mathrm{Y}$ connection are as follows:

(1) It is possible to bring out a lead from the neutral point of the winding, which is useful for various purposes.

(2) The cost is less than with the delta connection, requiring approximately 58 per cent of the turns.

(3) It is not possible for circulating currents of triple frequency to flow in the windings.

The delta connection does not appear to have any advantages except as a convenience in design for certain voltages. When used, proper precautions must be exercised in the design to eliminate excessive circulating currents.

THE FLEXIBILITY in design of three-phase machines,
as compared with two-phase, lies in the possibility of using either the $\mathrm{Y}$ or delta connection.

It might be expected that either connection would be used indiscriminately, depending on which worked out best for the case in hand.

However, it is probably safe to say that over ninety per cent of the three-phase generators built are $\mathrm{Y}$-connected.

The other cases are those in which, due to limitations in design as regards specified output, voltage etc., the delta connection permits the use of standard frames and tools, thus avoiding development. A few cases will also arise where customer's specifications call for the delta connection.

Some of the advantages of the $\mathrm{Y}$ connection are as follows:

(1) The possibility of bringing out a lead for various uses from the neutral of the winding.

This lead may be grounded. It may be used as the return in a four-wire system, giving with a 240 -volt generator, the voltage between the neutral and the end of each phase for incandescent lighting; similarly, on a 4000-volt generator, giving 2300 volts for use with standard transformers for three-phase four-wire 
systems of distribution. In this latter case the neutral is generally grounded. This system is used in many large cities.

(2) The $\mathrm{Y}$ connection gives the required voltage with 58 per cent of the turns required by the delta connection. This is a large factor in cost; especially in machines of high voltage and large numbers of turns per coil, where the expense of internal instulation increases rapidly.

(3) In a Y-connected machine, with ungrounded neutral, it is impossible for currents of triple frequency or multiples of triple frequency to flow, since the third harmonics, existing in the two legs of the $\mathrm{Y}$ winding which are in series between a pair of terminals, are opposed to cach other and of equal value, thus neutralizing one another.

In a delta-connected machine, the triple harmonics are in phase with each other, and will send triple frequency currents through the three phases in series of the closed delta. These currents are internal in the windings, the e.m.f. which causes them to flow being short-circuited in the closed delta, not appearing in the e.m.f. measured between terminals.

These circulating currents may be of great magnitude, entailing large $I^{2} R$ losses in the windings and corresponding loss of efficiency.

(4) In general, for the average alternator, the e.m.f. wave of the $\mathrm{Y}$-connected winding is nearer a true sine than that of the delta-connected gencrator.

(5) The use of the grounded ncutral reduces the potential strain on the insulation of the windings, permitting reduced thickness of insulation.

The above indicate the merits of the $\mathrm{Y}$ connection.

On the other hand the delta connection does not appear to have any advantages. When used, care must be exercised in design, paying attention to the percentage of pole-face width to pole pitch, shape of pole face, saturation of the magnetic circuit, etc.

Fig. 1 gives the wave shape of the e.m.f. on closed delta of a 900-kv-a., 25-cycle, 400-volt unit, in which it was found that the circulating current at excitation for no-load full voltage was 83 per cent of full load current.

At 75, 87.5, 100 and 112.5 per cent of full voltage no-load, the circulating current was found to be 59,69, 83 and 107 per cent, respectively, of full load current. 
PLATE XXXVIII

A. I, E. E.

VOL. $X X X I I I$, NO. 5

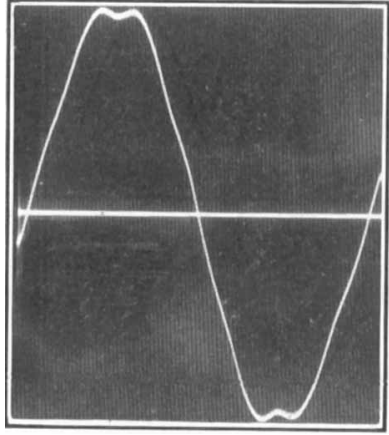

[EDEN]

Fig. 1-Potential Wave of 900KV-A. 25-CyCle, 400-VOLT GENerator, Closed “Delta ”.

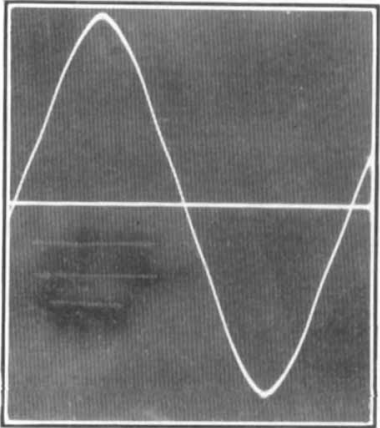

[EDEN]

Fig. 2-Potential Wave of 90()KV-A., 25-CyCle, 400-Volt GENERATOR, OPEN " DELTA ".

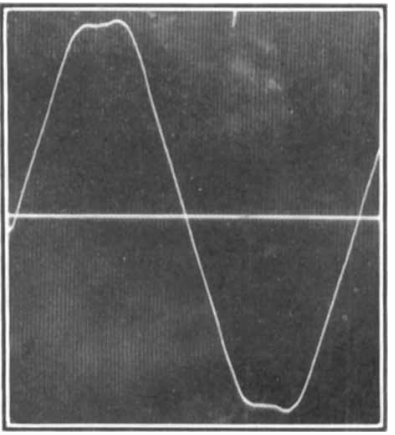

[EDEN]

Fig. 3-Potential Wave of 7.5-kV-A., 60-Cycle, 110-Volt Generator, Closed "Delta".

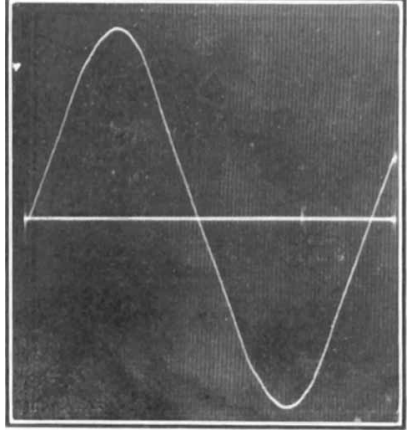

[EDEN]

Fig. 4-Potential Wave of 7.5KV-A., 60-CyCle, 110-VOLT GENERATOR, OPEN " Delta".

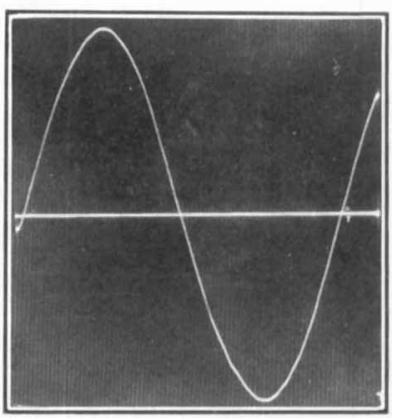

|EDEN|

Fig. 5-Potential Wave of 7.5KV-A., 60-CYCLE, 110-VOLT GENERATOR, ACROSS TERMINALS CONNECTED Y. 

The analysis of this wave shape shows the equation of the curve to be

$$
\begin{aligned}
E= & +99.9 \sin (\alpha-0.01 \text { deg. }) \\
& +1.0 \sin (5 \alpha+36 \mathrm{deg} .) \\
& -1.8 \sin (5 \alpha+4 \mathrm{deg} .) \\
& +2.9 \sin (7 \alpha-16 \mathrm{deg} .) \\
& +0.2 \sin (9 \alpha+59 \mathrm{deg} .) \\
& -1.1 \sin (11 \alpha+72 \mathrm{deg} .) \\
& -0.3 \sin (13 \alpha-45 \mathrm{deg} .)
\end{aligned}
$$

Similarly, Fig. 2 shows the wave shape of c.m.f. taken across one leg of the delta, one corner of the delta being open.

The analysis of this curve gives the equation

$$
\begin{aligned}
E= & 99.9 \sin (\alpha-0.1 \mathrm{deg} .) \\
& -5.9 \sin (3 \alpha-9 \mathrm{deg} .) \\
& +0.3 \sin (5 \alpha+28 \mathrm{deg} .) \\
& -0.1 \sin (7 \alpha-86 \mathrm{deg} .) \\
& +1.1 \sin (9 \alpha+8 \mathrm{deg} .) \\
& +0.1 \sin (11 \alpha+\mathrm{deg} .) \\
& +0 \sin (13 \alpha+0 \mathrm{deg} .)
\end{aligned}
$$

In the former the percentage of third harmonic is small as compared with the latter, the e.m.f. being used up in overcoming the internal impedance of the machine. However the fifth and seventh are strong, being produced by the pulsations of the field set up by the circulating current in the delta. The latter shows a third harmonic approximately 6 per cent of the fundamental, the others being practically negligible.

The $\mathrm{Y}$ connection constructed by adding two waves like Fig. 2, displacing them 60 deg., gives practically a sine curve. The use of a $\mathrm{Y}$ connection in this instance would have effected a saving of over 1 per cent in efficiency at full load.

Similarly, curves in Figs. 3, 4 and 5 show e.m.f. waves of closed delta, open delta and $\mathrm{Y}$ connection on a $7.5-\mathrm{kw}$. alternator in which the circulating current was a large percentage of full load current.

A further example of the effect of saturation on circulating current is seen in the following table, made up from tests of a 3000-kv-a., 6600-volt, 112 rev. per min., 60-cycle generator, connected delta for testing purposes. 


$\begin{array}{rl}57 & \\ 77 & 6 \\ 87 & 7.7 \\ 94 & 8 \\ 107 & 7.7 \\ 115 & 3 \\ \end{array}$

By using a $66_{3}^{2}$ per cent pitch winding, with the delta connection, the third and multiples of the third harmonic are eliminated. (See Trans. A. I. E. E., Vol. 28 (1909) page 1064.)

Thus in a $2500-\mathrm{kv}$-a., 2300-volt, 133-rev. per min., 60-cycle generator delta-connected, using $66_{3}^{2}$ per cent pitch, an ammeter

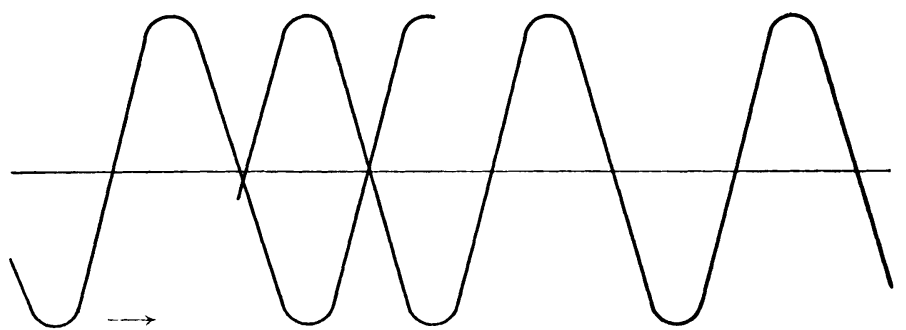

Fig. 6-Potential Wave of 2500-kV-A., 60-Cycle, 2300-Volt Gen erator, Open Delta.

in the corner of the delta gave no reading. The wave shape taken across one leg of the winding, with the delta open, is shown in Fig. 6.

It would therefore seem that from the standpoint of the designer, the $\mathrm{Y}$ connection is the safest to use and that great care must be exercised in using the delta connection. 